\title{
Effects of root zone cooling on butterhead lettuce grown in tropical conditions in a coir- perlite mixture
}

\begin{abstract}
Growing temperate vegetable crops such as lettuce in the humid tropical climate of lowland Malaysia commonly results in heat-induced crop damage, delayed heading, and precocious bolting. Manipulating the root zone to a cooler temperature may alleviate plant damage caused by high ambient temperatures to a large extent. In this study, the effects of root zone cooling (RZC) on the growth and development of butterhead lettuce were studied in a containerized planting system containing a 3:1 coir-perlite mixture. Polyvinyl chloride (PVC) pipes were used to circulate cold water and cool the root zone of the experimental plants, while their shoots were exposed to the hot ambient temperature. The temperature of the root zone was measured at different depths $(50 \mathrm{~mm}, 100 \mathrm{~mm}, 150 \mathrm{~mm}$, and $200 \mathrm{~mm}$ from the surface of the media). The butterhead lettuce canopy diameter and number of leaves were significantly higher in the RZC treatment at the end of the growth stage compared to the control plants, and the yield, as measured by the shoot and root dry weight, as well as the root/total biomass ratio were also improved. The cooling pipes placed $100 \mathrm{~mm}$ below the surface provided a better growth condition for butterhead lettuce; at $100 \mathrm{~mm}$, the RZC media had a mean daily temperature of $19^{\circ} \mathrm{C}$ and a minimum temperature of $14.4^{\circ} \mathrm{C}$ at $18: 00$. This study demonstrated the importance of optimizing root temperature for butterhead lettuce in an ambient hot tropical climate.
\end{abstract}

Keyword: Root zone cooling; Butterhead lettuce; Tropical condition; Coir-perlite mixture 